\title{
Towards a unified description of total and diffractive structure functions at DESY HERA in the QCD dipole picture
}

\author{
A. Bialas \\ Institute of Physics, Jagellonian University, Reymonta 4, 30-059 Cracow, Poland \\ R. Peschanski \\ Commissariat à l'Energie Atomique, Service de Physique Théorique, CE-Saclay, F-91191 Gif-sur-Yvette Cedex, France \\ Ch. Royon \\ Commissariat à l'Energie Atomique, DAPNIA, Service de Physique des Particules, CE-Saclay, F-91191 Gif-sur-Yvette, France
}

(Received 1 December 1997; published 4 May 1998)

\begin{abstract}
It is argued that the QCD dipole picture allows us to build a unified theoretical description, based on Balitskii-Fadin-Kuraev-Lipatov dynamics, of the total and diffractive nucleon structure functions. This description is in qualitative agreement with the present collection of data obtained by the H1 Collaboration. More precise theoretical estimates, in particular the determination of the normalizations and proton transverse momentum behavior of the diffractive components, are shown to be required in order to reach definite conclusions. [S0556-2821(98)03011-2]
\end{abstract}

PACS number(s): 13.60.Hb, 12.38.Bx

\section{MOTIVATION}

Considering the phenomenological discussion on the proton structure functions measured by deep-inelastic scattering of electrons and positrons at the DESY ep collider HERA, it is striking to realize that the proposed models, on one side for the total quark structure function $F_{2}\left(x, Q^{2}\right)[1]$ and on the other side for its diffractive component $F_{2}^{D(3)}\left(x, M^{2}, Q^{2}\right)[2]$, are in general distinct. Indeed, the models [3] aiming at the description of $F_{2}\left(x, Q^{2}\right)$ use a QCD-inspired "hard Pomeron' parametrization related either to a DokshitzerGribov-Lipatov-Altarelli-Parisi (DGLAP) [4] evolution with extrapolation at small $x$ [5] or to Balitskii-Fadin-KuraevLipatov (BFKL) [6] dynamics. On the other hand, most of the models proposed for the diffractive component of the quark structure function rely on a "soft Pomeron" picture of diffraction, assuming a point-like structure of the Pomeron considered as a compound particle $[7,8]$.

It has been known for some time, however, that at high energies the elastic scattering and diffraction dissociation of hadrons are closely related [9], being both a reflection of the same phenomenon, namely absorption of the incident particle wave in the target. It seems therefore interesting to verify if the same applies also to the incident virtual photons.

In the present paper we investigate this question in the framework of the QCD dipole picture $[10,11]$. This picture turned out already to be successful in the description of the total virtual photon-nucleon cross section (i.e., of total nucleon structure function $F_{2}$ [12]). The purpose of the present paper is to verify if the so-called rapidity gap events [3] discovered recently at HERA can also be described along these lines. Diffractive dissociation of the virtual photons in the framework of the QCD dipole picture was recently discussed in $[13,14]$. It was argued that the diffractive cross section consists of two components.

Component I. The inelastic component when the gluon cascade which evolved from the incident virtual photon interacts inelastically with the target, see Fig. 1(a). This component (corresponding to the 3-Pomeron interaction in the Regge terminology) contributes mainly to the region of very large mass $M$ of the diffractively excited system: $\beta \ll 1$, where, as usual, $\beta=Q^{2} /\left(Q^{2}+M^{2}\right)$.

Component II. The quasielastic component when the $q \bar{q}$ pair emerging from the virtual photon scatters elastically from the target, see Fig. 1(b). This component contributes to the region of smaller masses $\beta \geqslant 0.2$.

The model calculations of Refs. $[13,14]$ provided the formulas for differential cross section $d \sigma / d M^{2}$ of both components I and II. Unfortunately, for technical reasons, some rather drastic assumptions had to be made.

(a) The calculations were performed in the limit of large impact parameters. The integrated cross section was then estimated by integration only up to a certain cutoff $b_{\min }$. This procedure leads to a serious underestimate of the cross section [15].

(b) The target nucleon was treated as a collection of several QCD dipoles all of the same size and sitting at one point.

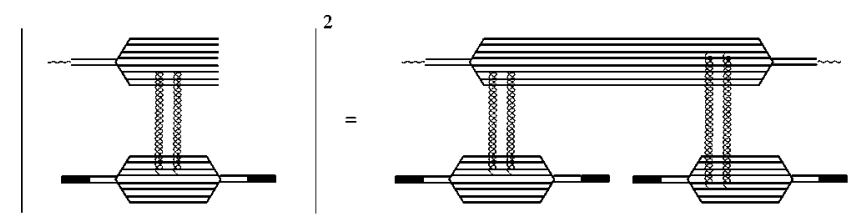

(a)

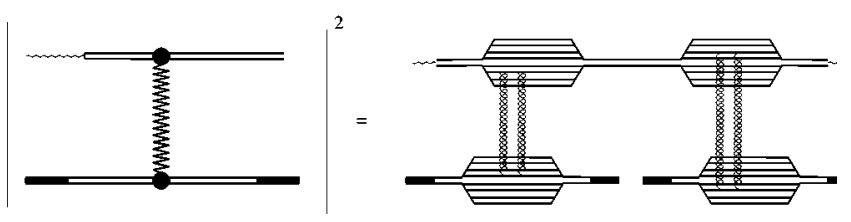

(b)

FIG. 1. (a) Inelastic diffraction (component I); (b) Quasielastic diffraction (component II). 
This assumption neglects the effects of nucleon form-factor and thus leads to an overestimation of the cross sections.

We are thus led to the conclusion that there are at present no reliable predictions for the absolute normalization of the diffractive cross sections of the virtual photons. In this situation, in order to compare the predictions with the data, we decided to treat the normalization constants in the two components as arbitrary parameters and restrict ourselves to the comparison of the observed dependence on kinematic variables to that predicted by the formulas of Refs. $[13,14]$ (cf. also [16]). Our work should thus be treated as an exploratory search which is a guide for further investigation and should be repeated once more reliable calculations are available. Within these caveats our investigation leads to the conclusion that the data on rapidity gap events published recently by H1 Collaboration [2] are reasonably well described by the QCD dipole picture and thus the Good-Walker idea seems consistent with these data.

The plan of our investigations is as follows. In the next section we remind briefly the QCD dipole picture results for the total photon-nucleon cross section, introduce the necessary notation, and perform a fit for the total structure function. In Sec. III we summarize the formulas for diffractive cross section of the components (I) and (II). These results are compared to the data [2] in Sec. IV. Finally Sec. V contains our conclusions, as well as an outlook for further work.

\section{PROTON STRUCTURE FUNCTIONS}

In the QCD-dipole picture of high-energy scattering of two initial small-size $(r, \bar{r})$ onia (massive $q \bar{q}$ states), the total cross section at fixed impact parameter $\sigma(b)$ can be obtained from the all-order QCD resummation of the elementary dipole-dipole cross sections $\sigma(\rho, \bar{\rho})$, where dipole states of transverse diameter $\rho$ (respectively, $\bar{\rho}$ ) appear in the wave function of the initial states of transverse diameter $r$ (respectively, $\bar{r}$ ) at the "time" of interaction. This "time" variable is represented by a rapidity variable $\ln c / \xi$, where $c$ is a phenomenological constant [17] and $\xi$ is the Bjorken variable labelling the softer end of the produced dipole. One writes

$$
\begin{aligned}
\sigma_{t o t} & =\int d^{2} b \sigma(b) \\
& =\int \frac{d \rho}{\rho} n_{1}(r ; \rho, \xi) \int \frac{d \bar{\rho}}{\bar{\rho}} n_{1}(\bar{r}, \bar{\rho}, \bar{\xi}) \sigma(\rho, \bar{\rho}),
\end{aligned}
$$

where the partition of the total "time" $\ln c / x=\ln c / \xi \bar{\xi}$ between the target and projectile is arbitrary, provided $\xi \bar{\xi}=x$. $n_{1}(r, \rho, \xi)$ is the multiplicity of dipoles of size $\rho$, integrated over the transverse distance from the center of the onium, generated from an initial dipole of size $r$ after a "time" $\ln c / \xi$. It is given by

$$
n_{1}(r, \rho, \xi)=\int \frac{d \gamma}{2 \pi i}\left(\frac{r}{\rho}\right)^{\gamma} e^{\Delta(\gamma) \ln (1 / \xi)},
$$

where

$$
\Delta(\gamma)=\frac{\alpha N}{\pi}[2 \psi(1)-\psi(1-\gamma / 2)-\psi(\gamma / 2)]
$$

is the eigenvalue of the BFKL kernel [6], and $N=3$ is the number of colors.

The elementary dipole-dipole cross sections are obtained from the gluon-exchange graphs and give [10]

$$
\begin{aligned}
\sigma(\rho, \bar{\rho}) & =8 \pi \alpha^{2} \int \frac{d \ell}{\ell^{3}}\left[1-J_{0}(\ell \rho)\right]\left[1-J_{0}(\ell \bar{\rho})\right] i \\
& =2 \pi \alpha^{2} \rho_{<}^{2}\left[1+\ln \left(\rho_{>} / \rho_{<}\right)\right] .
\end{aligned}
$$

Inserting formulas (2) and (4) in the cross section formula (1), one finds

$$
\sigma(r, \bar{r}, \xi)=2 \pi \alpha^{2} r \bar{r} \int \frac{d \gamma}{2 \pi i}\left(\frac{r}{\bar{r}}\right)^{\gamma-1} \frac{4}{\gamma^{2}(2-\gamma)^{2}} e^{\Delta(\gamma) \ln (1 / \xi)} .
$$

In order to obtain the virtual photon-proton cross section from Eq. (5), one has to integrate over the initial distributions of dipoles inside the photon and the proton. Since we know neither the number nor the distribution of the dipoles in the proton, we simply define

$$
\int d^{2} \bar{r}(\bar{r})^{2-\gamma} \Phi(\bar{r}) \equiv n_{e f f}(\gamma)\left[r_{0}(\gamma)\right]^{2-\gamma},
$$

where $n_{\text {eff }}$ has the meaning of the average number of primary dipoles in the proton and $r_{0}$ is their average transverse diameter.

The distributions of the primary dipoles in the virtual photons are known $[18,11]$ and thus the corresponding integrals can be performed with the result

$$
\begin{aligned}
\sigma_{T, L}\left(x, Q^{2}\right)= & \frac{4 N \alpha_{e m} e_{f}^{2}}{\pi} \alpha^{2} n_{e f f} \int \frac{d \gamma}{2 \pi i} r_{0}^{2}\left(\frac{2}{Q r_{0}}\right)^{\gamma} \\
& \times e^{\Delta(\gamma) \ln (1 / x)} \frac{4}{\gamma^{2}(2-\gamma)^{2}} \\
& \times \frac{\Gamma^{2}(2-\gamma / 2) \Gamma^{4}(1+\gamma / 2)}{\Gamma(4-\gamma) \Gamma(2+\gamma)} H_{T, L}(\gamma),
\end{aligned}
$$

where

$$
H_{T}(\gamma)=\frac{(2-\gamma / 2)(1+\gamma / 2)}{\gamma(1-\gamma / 2)}, \quad H_{L}(\gamma)=1 .
$$

$H_{T, L}$ refers to transverse and longitudinal photons, respectively. $e_{f}^{2}$ is the total charge of the quarks whose flavor contributes to the reaction, and $n_{e f f}=n_{e f f}(1), \quad r_{0}=r_{0}(1)$, see formula (6).

The path integral in Eq. (7) can be evaluated by the saddle point method (giving good approximation as $x \rightarrow 0$ ). The result is

$$
F_{T, L}=\frac{Q^{2}}{4 \pi^{2} \alpha_{e m}} \sigma_{T, L}
$$




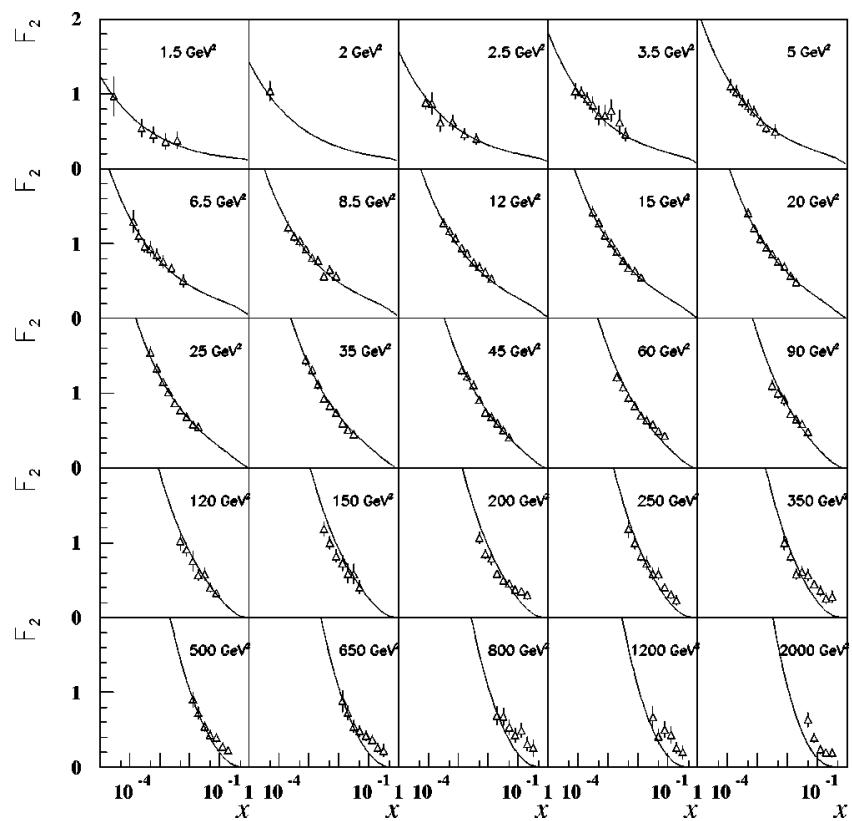

FIG. 2. Comparison of the 4-parameter fit with the H1 data. The validity of the prediction extends beyond the domain included in the fit. We note a discrepancy at high $x$, high $Q^{2}$ due, in particular, to the absence of the valence contribution not considered in the present model.

$$
\begin{aligned}
= & H_{T, L}(1) \frac{\pi N \alpha^{2} e_{f}^{2}}{32} n_{e f f}\left(\frac{x}{c}\right)^{-\Delta_{P}} \frac{r_{0} Q}{2}\left(\frac{2 a(x)}{\pi}\right)^{1 / 2} \\
& \times \exp \left[-\frac{a(x)}{2} \ln ^{2}\left(\frac{r_{0} Q}{2}\right)\right],
\end{aligned}
$$

where $H_{L}(1)=1, \quad H_{T}(1)=9 / 2$, and

$$
a(\xi)=[7 \alpha N \zeta(3) \ln (c / \xi) / \pi]^{-1}, \quad \Delta_{P} \equiv \Delta(1)=4 \ln 2 \alpha N / \pi
$$

are the well-known coefficients appearing in the solution of BFKL dynamics for the Pomeron [6].

Formula (9) gives the prediction for the nucleon structure functions in terms of four parameters: the strong coupling constant $\alpha$, the average number of primary dipoles in the proton $n_{\text {eff }}$, their average radius $r_{0}$, and the constant $c$ fixing the rapidity scale of the problem. It coincides with the one used in the published fit [12] apart from the new parameter $c$ which sets the rapidity scale of the process, and is unavoidable in the leading log approximation of QCD. This justifies a new fit of $F_{2}$ using formula (9) which we have performed assuming $\Delta_{P}=0.282$ (as in [12]) and leaving free the three other parameters. The result is

$$
\begin{gathered}
\Delta_{P}=0.282, \quad c=1.75 \\
Q_{0}=\frac{2}{r_{0}}=0.622 \mathrm{GeV}, \quad n_{e f f}=3.8 / e_{f}^{2} .
\end{gathered}
$$

The fit (displayed in Fig. 2) is using the published data from the $\mathrm{H} 1$ experiment [1]. We have only considered the points with $Q^{2} \leqslant 150 \mathrm{GeV}^{2}$ to remain in a reasonable domain of validity of the QCD dipole model. Changing this value does not appreciably change the quality and parameters of

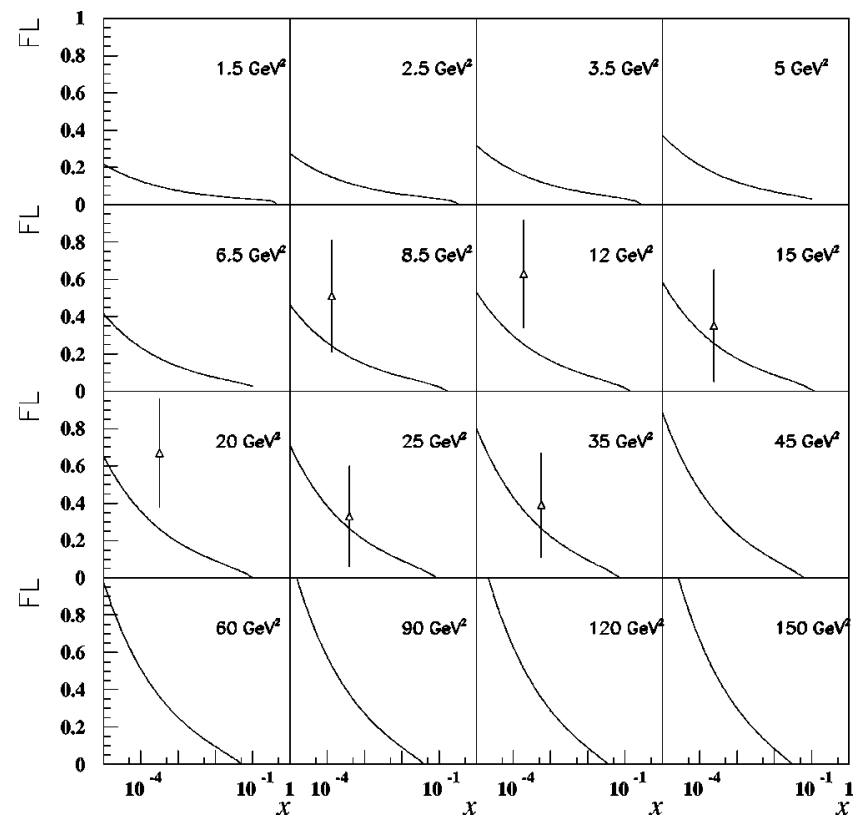

FIG. 3. Comparison of our prediction for the longitudinal structure function $F_{L}$ and the $\mathrm{H} 1$ data. The prediction is somewhat lower than the measurement, but more precise data are needed to make more precise tests.

the fit. The $\chi^{2}$ is 88.7 for 130 points. Although not included in the fit, the data points at high $Q^{2}>150 \mathrm{GeV}^{2}$ and $x$ $<5.10^{-1}$ are well described, while at higher $x$, an expected contribution of valence quarks is needed.

Commenting on the parameters, let us note that the effective coupling constant extracted from $\Delta_{P}$ is $\alpha=0.11$, close to $\alpha\left(M_{Z}\right)$ used in the H1 QCD fit. It is an acceptable value for the small coupling constant required by the BFKL framework. ${ }^{1}$ The value of $Q_{0}$ corresponds to a transverse radius of $0.4 \mathrm{fm}$ which is in the correct range for a proton nonperturbative characteristic scale. The value of $n_{\text {eff }}$ determines the number of primordial dipoles in the proton to be about 6 (if three flavors contribute to the process) which also does not seem unreasonable. The parameter $c$ sets the "time' scale for the formation of the interacting dipoles. It defines the effective total rapidity interval which is $\ln (1 / x)$ $+\ln c$, the constant being not predictable (but of order 1) at the leading logarithmic approximation.

The obtained fit for $F_{2}$ is very similar than the previously published one in Ref. [12], even with a better $\chi^{2}$. In the same spirit, relation (11) provides a parameter-free prediction for the gluon density (not shown in the figures) which is, as the previous one [12], in good agreement with the results obtained by the H1 QCD fits based on a next leading order (NLO) DGLAP evolution equation [1]. Using the factorization properties of formula (11) and noting [12] that the $F_{L}$ structure function is given by a similar formula with $h_{T}$ $+h_{L}$ replaced by $h_{L}$, one obtains a parameter-free prediction for $F_{L}$ (see Fig. 3). Note that we obtain a prediction in agree-

\footnotetext{
${ }^{1}$ The running of the coupling constant and other next leading log corrections are not taken into account in the present BFKL scheme. This could explain the rather low value of the effective $\Delta_{P}$ which is expected to be decreased by the next leading contributions [19].
} 
ment with the (indirect) experimental determination for $F_{L}$ [20], but somewhat lower than the center values. Thus, it would be interesting to obtain a more precise measurement of $F_{L}$ to test the different predictions on the $Q^{2}$ evolution as already mentioned in Ref. [12].

\section{DIFFRACTIVE STRUCTURE FUNCTIONS}

The diffractive structure functions are related to the corresponding diffractive $\gamma^{*}$-nucleon cross sections by the relation

$$
F_{T, L}^{D(3)}\left(Q^{2}, x_{P}, \beta\right) \equiv \frac{Q^{2}}{4 \pi^{2} \alpha_{\text {e.m. }}} x_{P}^{-1} \int d^{2} b \frac{\beta d \sigma_{T, L}}{d \beta d^{2} b},
$$

where $x_{P}=x / \beta$.

As already explained in the first section, in the QCD dipole model the diffractive structure functions are given by two components: inelastic and quasielastic. They were discussed in $[13,14]$, where the formulas for $\gamma^{*}$-dipole diffractive cross sections were derived and used to construct the corresponding structure functions following the formula. These results are summarized below.

(I) Inelastic component:

$$
\begin{aligned}
F_{T, L}^{D(3), \text { inel }}\left(Q^{2}, x_{P}, \beta\right) & \\
= & \frac{16 e_{f}^{2} \alpha^{5} N}{\pi} n_{e f f}^{2}\left(\frac{2 a\left(x_{P}\right)}{\pi}\right)^{3} x_{P}^{-1-2 \Delta_{P}} \\
& \times \int_{c-i \infty}^{c+i \infty} \frac{d \gamma}{2 \pi i}\left(\frac{r_{0} Q}{2}\right)^{\gamma} \Omega(\gamma) H_{T, L}(\gamma) \beta^{-\Delta(\gamma)},
\end{aligned}
$$

where $H_{T, L}$ are defined in Eq. (8),

$$
\Omega(\gamma)=V(\gamma) \frac{2}{\gamma(2-\gamma)^{3}} \frac{\Gamma^{4}(2-\gamma / 2) \Gamma^{2}(1+\gamma / 2)}{\Gamma(4-\gamma) \Gamma(2+\gamma)}
$$

and

$$
V(\gamma)=\int_{0}^{1}{ }_{2} F_{1}\left(1-\gamma, 1-\gamma ; 1 ; y^{2}\right) d y
$$

$\left({ }_{2} F_{1}\right.$ is the hypergeometric function). In the interesting 3pomeron limit $(\beta \ll 1)$, the path integral can be evaluated by the saddle point method with the result

$$
\begin{aligned}
& F_{T, L}^{D(3), \text { inel }}\left(Q^{2}, x_{P}, \beta\right) \\
&= G H_{T, L}(1) \frac{e_{f}^{2} \alpha^{5} N^{2} \pi}{4}\left(\frac{2 a\left(x_{P}\right)}{\pi}\right)^{3} \\
& \times x_{P}^{-1-2 \Delta_{P}} \frac{r_{0} Q}{2} \beta^{-\Delta_{P}}\left(\frac{2 a(\beta)}{\pi}\right)^{\frac{1}{2}} \\
& \times \exp \left(-\frac{a(\beta)}{2} \ln ^{2}\left(r_{0} Q / 2\right)\right),
\end{aligned}
$$

where $G=0.915 \ldots$ is Catalan's constant, $H_{T}(1)$ $=9 / 2, \quad H_{L}(1)=1$.
The important features of Eq. (16), pointed out in [13] are as follows.

(a) An approximate factorization of the $x_{P}$ and $Q^{2}$ dependences.

(b) Important logarithmic corrections of the form $\left[\ln \left(1 / x_{P}\right)\right]^{-3}$ to the main power law factor $x_{P}^{-1-2 \Delta_{P}}$. These corrections lower the effective pomeron intercept for diffractive dissociation, in qualitative agreement with the data.

(c) There is a significant scaling violation, because $F_{T, L}^{D(3)}$ depends explicitly on $Q^{2}$.

(II) Quasielastic component.

It was discussed in [14], where the formulas for diffractive cross sections in $\gamma^{*}$-dipole collisions were given. From these formulas one can derive the following expression for the diffractive structure functions:

$$
\begin{aligned}
& F_{T}^{D(3), q e l}\left(Q^{2}, x_{P}, \beta\right) \\
& =\frac{Q^{4} N_{c} e_{f}^{2}}{2 \pi^{3} \beta x_{P}} n_{e f f}^{2} \int_{r_{0}}^{\infty} d^{2} b \int_{0}^{1} d z\left[z^{2}+(1-z)^{2}\right] z^{2}\left(1-z^{2}\right) \\
& \quad \times\left|\int_{0}^{r_{0}} d \rho T\left(b, \rho, r_{0}, \xi\right) K_{1}(\hat{Q} r) J_{1}(\hat{M} r)\right|^{2}
\end{aligned}
$$

and

$$
\begin{aligned}
& F_{L}^{D(3), q e l}\left(Q^{2}, x_{P}, \beta\right) \\
& =\frac{Q^{4} N_{c} e_{f}^{2}}{\pi^{3} \beta x_{P}} n_{e f f}^{2} \int_{r_{0}}^{\infty} d^{2} b \int_{0}^{1} d z z^{3}(1-z)^{3} \\
& \quad \times\left|\int_{0}^{r_{0}} d \rho T\left(b, \rho, r_{0}, \xi\right) K_{0}(\hat{Q} r) J_{0}(\hat{M} r)\right|^{2},
\end{aligned}
$$

where

$$
\hat{Q}^{2}=z(1-z) Q^{2}, \quad \hat{M}^{2}=z(1-z) M^{2},
$$

and $T\left(b, \rho, r_{0}, x_{P}\right)$ is the amplitude for elastic scattering of a dipole of diameter $\rho$ on a dipole of diameter $r_{0}$ at impact parameter $b$.

In Ref. [14] this amplitude was approximated by its asymptotic form valid for large $b$ which reads

$$
\begin{aligned}
& T\left(b, \rho, r_{0}, x_{P}\right) \\
& \quad \approx \pi \alpha^{2} \frac{\rho r_{0}}{b^{2}} \ln \left(\frac{b^{2}}{\rho r_{0}}\right) x_{P}^{-\Delta_{P}}\left(\frac{2 a\left(x_{P}\right)}{\pi}\right)^{3 / 2} e^{a\left(x_{P}\right) / 2} \ln ^{2}\left(\frac{b^{2}}{\rho r_{0}}\right)
\end{aligned}
$$

and for that reason the integration over $b$ was performed from $r_{0}$ to $\infty$ [the meaning of the formula (20) for $b<r_{0}$ is rather doubtful].

The main qualitative features of this quasielastic component, pointed out in [14], are (a) a similar $x_{P}$ dependence as the inelastic component, with important logarithmic corrections bringing down the pomeron intercept, (b) as expected, the quasielastic component vanishes at $\beta=0$ and actually populates significantly only the region $\beta \geqslant 0.2$, and (c) the dependence on $\beta$ of the transversal and longitudinal structure 


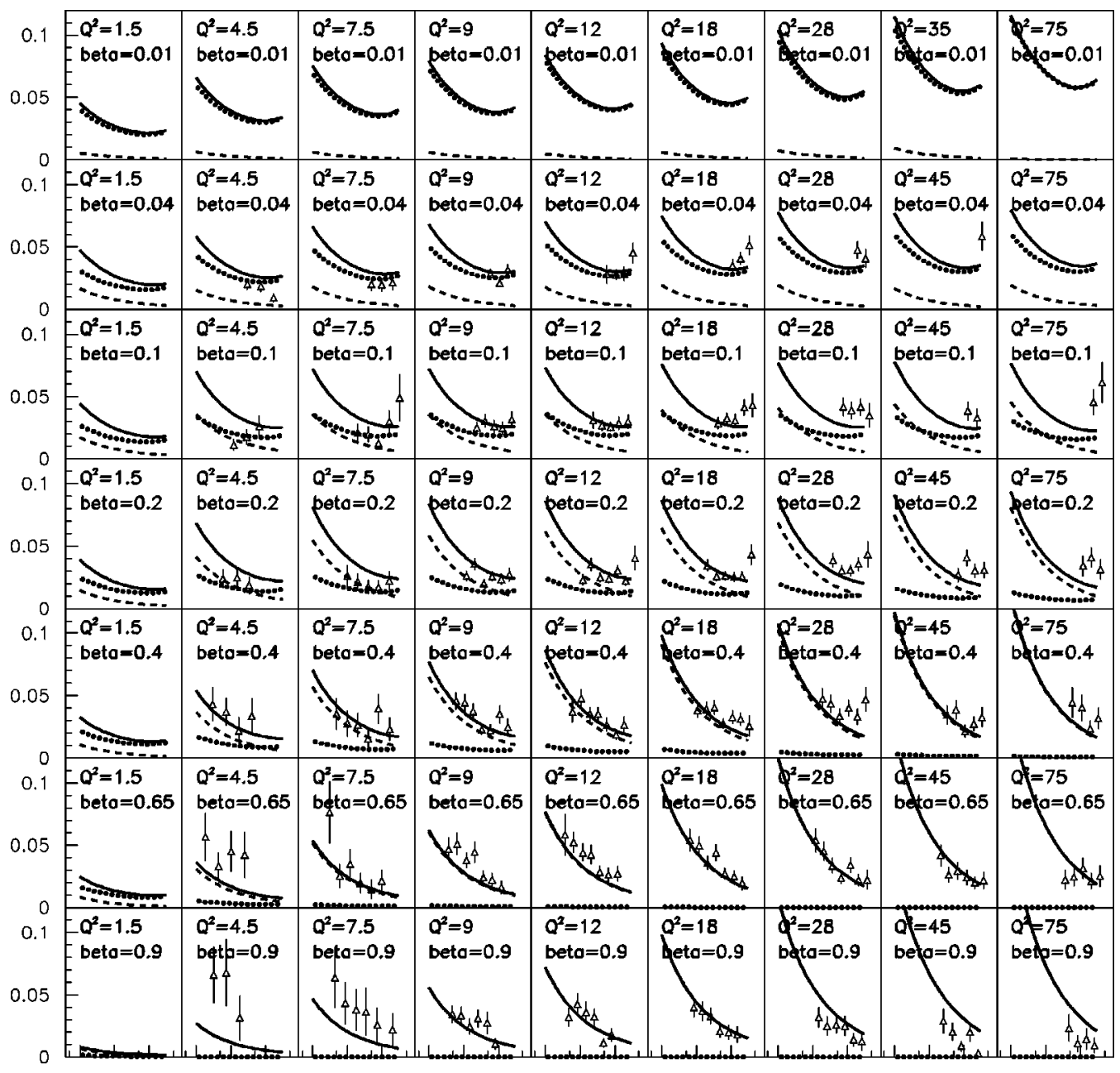

FIG. 4. Prediction for the total (longitudinal + transverse) diffractive structure function, see text. Dotted lines: the inelastic component I; Dashed lines: the quasielastic component II; Full line: the sum of both components (note that at $\beta \approx 1$, the inelastic component is almost 0 and the dashed line coincides with the full line and thus is not apparent on the plot).

functions is dramatically distinct. $F_{T}$ dominates in the region $\beta \leqslant 0.8$, whereas $F_{L}$ takes over at small $\beta$. The sum of the two components, however, is almost constant in the range $0.3 \leqslant \beta<1$.

\section{PREDICTIONS FOR HARD DIFFRACTION}

The formulas presented in Sec. IV were obtained in $[13,14]$ by calculating first the cross section of $\gamma^{*}$ on a single dipole of a fixed transverse diameter $r_{0}$ in the limit of very large impact parameter $b$. The obtained formulas were then extrapolated until $b_{\text {min }}=r_{0}$ and integrated from $b_{\text {min }}$ to $\infty$. Finally the result was multiplied by $n_{e f f}^{2}$ to account for the number of the dipoles in the target nucleon (determined from the fit of the formula for $F_{2}$ to the data).

These approximations allowed to perform explicit calculations and to discuss the general behavior of diffractive structure functions $[13,14]$. They are, however, not valid in the important region, where the impact parameter $b$ is of the order of the size of the original dipoles [15] and therefore the results given in the formulas of Sec. III cannot be treated as precise predictions of the QCD dipole picture (i.e., of BFKL dynamics) for several reasons.
First, the asymptotic formula for large impact parameter ignores entirely the singularities of the dipole-dipole amplitudes, which become important, when the impact parameter is of the order of the size of the colliding dipoles. This defect leads to a serious underestimation in the normalization of the calculated cross sections. ${ }^{2}$ However, the conformal invariance of the BFKL dynamics [21,22] insures that the general dependence on kinematic variables remains-to a good approximation-unaffected.

Second, the cross section for scattering on a single dipole of the size $r_{0}$, even if multiplied by $n_{e f f}^{2}$, cannot be directly used for the estimation of the cross section on the nucleon target. The reason is twofold: (i) it is unlikely that all the primary dipoles in the nucleon are of the same size $r_{0}$ and thus the distribution of their sizes must be taken into account, (ii) the single-dipole cross section ignores entirely the distribution of the transverse position of the primary dipoles in the nucleon, i.e., it ignores the effects of the nucleon form-factor. Although these effects are not present in forward scattering

\footnotetext{
${ }^{2}$ It was recently shown in [15] that this factor may even well exceed 100.
} 
amplitudes (and therefore they do not influence the calculation of the total cross section), they largely determine the momentum transfer dependence and thus reduce significantly the cross section integrated over momentum transfer to the target nucleon.

To summarize, we note [15] two effects which were not included in the calculations given in $[13,14]$ and which are expected to affect substantially the normalization of the obtained diffractive structure functions. In this situation before a more precise calculation is available, we treat the normalization of the two components as free parameters, in order to phenomenologically evaluate the main conditions for a test of the unified description of proton structure functions. We thus compare the experimental data to the formula

$$
F_{2}^{D(3)}=N_{\text {inel }} F_{2}^{D(3), \text { inel }}+N_{q e l} F_{2}^{D(3), q e l},
$$

where $F_{2}^{D(3), \text { inel }}$ and $F_{2}^{D(3), q e l}$ are constructed from the formulas (16), (17), and (18) using $F_{2}=F_{L}+F_{T}$.

Since this procedure can at best be considered only as an exploratory search, we did not try to perform a fit, but simply tried a few values of $N_{\text {inel }}$ and $N_{q e l}$ to see if one can obtain a qualitative agreement of Eq. (21) to the data. In Fig. 4 the results of these calculations are shown for $N_{\text {inel }}=16$ and $N_{q e l}=6$. One sees that a general description of the data is quite reasonable for $x_{P} \leqslant 0.01$ except in the region of large $\beta$, where the $Q^{2}$ dependence of the quasielastic component is not fully adequate.

We find this result rather satisfactory, given the present status of the theoretical calculations. Thus - although the final answer must wait till more precise QCD dipole calculations are available-our tentative conclusion is that the existing data on rapidity-gap events do not rule out the BFKL dynamics as a correct description of the diffractive phenomena involving virtual photons. Indeed a decisive test will come along with more complete theoretical calculations, e.g., [15].

\section{CONCLUSIONS AND OUTLOOK}

In conclusion, we have shown that the BFKL dynamics, as represented by the QCD dipole picture, is in qualitative agreement with the three-dimensional data on rapidity-gap events being observed at HERA. Further theoretical work is needed, however, to arrive at more precise conclusions. In particular, it is necessary:

(a) to evaluate the $\gamma^{*}$ cross sections without the large- $b$ approximation used in $[13,14]$. The work on inelastic component was recently completed [15] and the quasielastic component will be available in the near future.

(b) Since the effects related to the nucleon form-factor are expected to influence significantly the results, a serious phenomenological discussion of the nucleon form-factor in the framework of the QCD dipole picture is required. More precise data on momentum transfer dependence of the diffractive structure functions would be of great help. ${ }^{3}$

We feel that this program is feasible and thus one may hope that a unified picture of the high-energy diffractive processes involving the virtual photons, based on BFKL dynamics, may indeed be constructed in the near future.

\footnotetext{
${ }^{3}$ The form-factor effects being unimportant for forward scattering, the measurements of diffraction dissociation at zero momentum transfer would of course bring an important information to the problem we consider. At this point one may notice that also the measurements of the virtual photon shadowing in nuclei (which depends mainly on forward diffractive amplitudes [23]) could provide another practical method to learn about the diffraction at zero momentum transfer.
}

[1] H1 Collaboration, S. Aid et al., Nucl. Phys. B470, 3 (1996).

[2] H1 Collaboration, C. Adloff et al., Z. Phys. C 76, 613 (1997).

[3] For a recent review, see J. Bluemlein, J. Huston, C. Royon, and R. Yoshida, "Summary of Working group I: hadron structure;', P. Newman, "Colour singlet exchange in ep interactions;'” D. Soper, "Diffraction in DIS and elsewhere," Summary talks given at the "DIS 97" conference, Chicago.

[4] G. Altarelli and G. Parisi, Nucl. Phys. B126, 298 (1977); V. N. Gribov and L. N. Lipatov, Sov. J. Nucl. Phys. 15, 438 (1972); 15, 675 (1972); Yu. L. Dokshitzer, Sov. Phys. JETP 46, 641 (1977).

[5] M. Glück, E. Reya, and A. Vogt, Z. Phys. C 53, 127 (1992); Phys. Lett. B 306, 391 (1993).

[6] V. S. Fadin, E. A. Kuraev, and L. N. Lipatov, Phys. Lett. 60B, 50 (1975); I. I. Balitsky and L. N. Lipatov, Sov. J. Nucl. Phys. 28, 822 (1978).

[7] G. Ingelman and P. Schlein, Phys. Lett. 152B, 256 (1985).

[8] A. Edin, G. Ingelman, and J. Rathsman, Phys. Lett. B 366, 371 (1996); Z. Phys. C 75, 57 (1997); W. Buchmüller and A. He- becker, Phys. Lett. B 355, 573 (1995); Nucl. Phys. B476, 203 (1996).

[9] M. L. Good and W. D. Walker, Phys. Rev. 120, 1857 (1960); H. I. Miettinen and J. Pumplin, Phys. Rev. D 18, 1696 (1978).

[10] A. H. Mueller, Nucl. Phys. B415, 373 (1994); A. H. Mueller and B. Patel, ibid. B425, 471 (1994); A. H. Mueller, ibid. B437, 107 (1995).

[11] See also, N. N. Nikolaev and B. G. Zakharov, Z. Phys. C 49, 607 (1991).

[12] H. Navelet, R. Peschanski, and Ch. Royon, Phys. Lett. B 366, 329 (1996); H. Navelet, R. Peschanski, Ch. Royon, and S. Wallon, ibid. 385, 357 (1996).

[13] A. Bialas and R. Peschanski, Phys. Lett. B 378, 302 (1996); A. Bialas, Acta Phys. Pol. B 27, 6 (1996).

[14] A. Bialas and R. Peschanski, Phys. Lett. B 387, 405 (1996).

[15] A. Bialas, H. Navelet, and R. Peschanski, Saclay Report No. T97/131, hep-ph/9711236 [Phys. Lett. B (to be published)].

[16] M. Genovese, N. N. Nikolaev, and B. G. Zakharov, Sov. Phys. JETP 81, 625 (1995); 81, 633 (1995). 
[17] A. Bialas, Acta Phys. Pol. B 28, 1239 (1997).

[18] J. Bjorken, J. Kogut, and D. Soper, Phys. Rev. D 3, 1382 (1971).

[19] G. Camici and M. Ciafaloni, Phys. Lett. B 412, 396 (1997).

[20] H1 Collaboration, Phys. Lett. B 393, 452 (1997).

[21] L. N. Lipatov, Zh. Éksp. Teor. Fiz. 90, 1536 (1986) [Sov.
Phys. JETP 63, 904 (1986)].

[22] H. Navelet and R. Peschanski, Nucl. Phys. B507, 353 (1997); H. Navelet and S. Wallon, Report No. SACLAY-SPHT-97023 [Nucl. Phys. B (to be published)].

[23] A. Bialas, W. Czyz, and W. Florkowski, Phys. Rev. D 55, 6830 (1997); A. Bialas and W. Czyz (unpublished). 